\title{
SOPHISTICATED METHODS FOR TISSUE PERFUSION EVALUATED
}

This paper focuses on image acquisition system designing for biomedical image enhancement. It introduces the possibilities of diagnostics and analysis of tissue via virtual instrumentation. The two dimensional images shot by the camera provide information about the surface properties and degradation of a real skin. The main idea is based on the human skin parameters measured for healthy and ill skin. The aforementioned access provides parameters evaluation by creating a structure model. The anomalies are recognized by this model.

\section{Introduction}

When the image is taken, the light conditions are very important. If the image was too dark or bright, the image details are not visible. An optimal light must be maintained from this reason.

Solid-state light dimmers work by varying the "duty cycle" (on/off time) of the full AC voltage that is applied to the lights being controlled. For example, if the voltage is applied for only half of each AC cycle, the light bulb will appear to be much less bright than when it gets the full AC voltage, because it gets less power to heat the filament. Solid-state dimmers use the brightness knob setting to determine at what point in each voltage cycle to switch the light on and off.

Image segmentation can use global grayscale thresholding, global color thresholding, local thresholding, and morphological segmentation. Image segmentation is the process of separating objects from the background and each other so that each object can be identified and characterized.

Thresholding segments an image into a particle region-which contains the objects under inspection-and a background region based on the pixel intensities within the image. The resulting image is a binary image.

Thresholding can be used to extract areas that correspond to significant structures in an image and to focus analysis on these areas.

Thresholding an image is often the first step in a variety of machine vision applications that perform image analysis on binary images, such as particle analysis, golden template comparison, and binary particle classification.

\section{Design of light regulation}

On the basis of simulations a wiring dimmer was designed. The power supply is made directly from the mains supply $230 \mathrm{~V}$ via input transformer [1]. Dimming feature is provided through one PWM channel, which generates pulses at the gate on a switching transistor [2].

The measurement card is generating impulses to the circuit. The protection and electrical separation of the measurement card from the power circuit is guaranteed through the opto coupler and driver.

The dimmer control performance is realized through virtual instrumentation. The proposed virtual instrumentation included a connection of high speed video camera applications and light microscope regulatory loop. This regulation is implemented by setting the optimal switching pulse width (duty cycle). The standard frequency pulse from the measurement card is about $25 \mathrm{kHz}$ to $50 \mathrm{kHz}$.

The optimal duty cycle choice is realized by the ongoing analysis of statistical parameters of the histogram of image acquisition [3], [4].

The mean position of the image histogram is an important indicator of proper lighting (Fig. 1). The optimal mean value is the case in the mid-range gray level (128 at $8 \mathrm{bpp})$.

The measured characteristic is the value of the high speed video camera frame $60 \mathrm{fps}$. Based on measured images histograms for used halogen lamp and fps, the optimal duty cycle range is approximately 20-30\% The histogram mean was about 124 , in duty cycle $25 \%$ and a value close to the optimum value.

\footnotetext{
* Libor Hargas, Dusan Koniar, Stanislav Stofan, Miroslav Hrianka

Department of Mechatronics and Eletronics, Faculty of Electrical Engineering, University of Zilina, Slovakia, E-mail: libor.hargas@fel.uniza.sk
} 

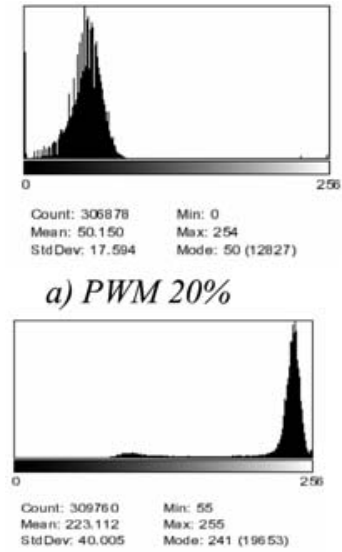

a) $P W M 30 \%$ a) $P W M 20 \%$

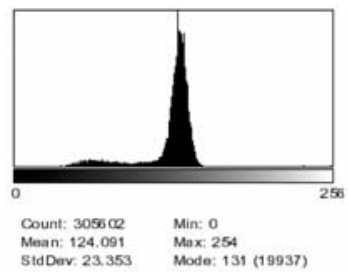

a) $P W M 25 \%$

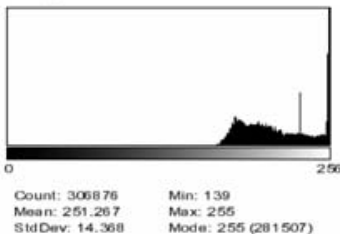

a) $P W M 40 \%$
Fig. 1 PWM image histograms

Frequency increase caused a shifting of duty cycle towards higher values. The halogen lamp lighting regulation is realized on the basis of the measured characteristic. The approximation curve is in Fig. 2. The duty cycle is changed with $1 \%$ resolution and it tried to set up mean value near value 128 . For different fps it is necessary to create different curves. The approximation for different fps is done by these curves.

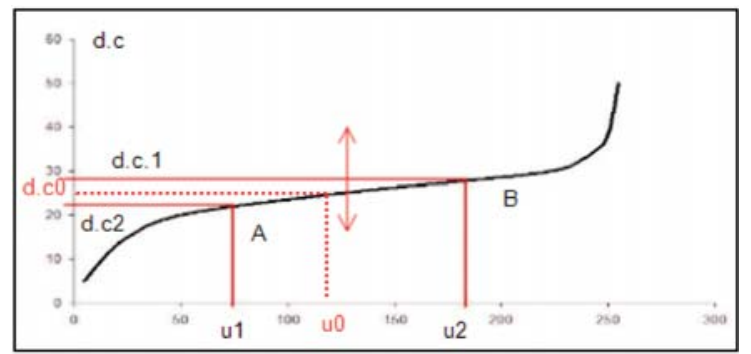

Fig. 2 Image histogram mean dependence on dimmer switching pulses duty cycle

We can approximate the curve between the points A and B (Fig. 2) as a linear line:

$$
\begin{aligned}
& y=a x+b \\
& A=\left\lfloor\mu_{1}, d \cdot c_{1}\right\rfloor ; \quad B=\left\lfloor\mu_{2}, d \cdot c_{2}\right\rfloor \\
& a=\frac{d \cdot c_{2}-d \cdot c_{1}}{\mu_{2}-\mu_{1}} \\
& b=d \cdot c_{1}-\frac{d \cdot c_{2}-d \cdot c_{1}}{\mu_{2}-\mu_{1}} \mu_{1} \\
& y=\frac{d \cdot c_{2}-d \cdot c_{1}}{\mu_{2}-\mu_{1}} x+d \cdot c_{1}-\frac{d \cdot c_{2}-d \cdot c_{1}}{\mu_{2}-\mu_{1}} \mu_{1}
\end{aligned}
$$

$$
\begin{aligned}
& \mu_{i}<\mu_{0} \Rightarrow d \cdot c \uparrow \\
& \mu_{i}>\mu_{0} \Rightarrow d \cdot c \downarrow \\
& d \cdot c_{i}-d \cdot c_{0}= \pm \Delta d \cdot c
\end{aligned}
$$

where $d \cdot c$ - duty cycle value

$$
\mu \text { - gray level value }
$$

The dimmer control performance is realized through virtual instrumentation.

\section{Image preprocessing}

\section{Global Grayscale Thresholding}

Global grayscale thresholding includes manual thresholding and automatic thresholding techniques. Global thresholding works best when the inspection images exhibit a uniform lighting both within each image and across multiple images. Particles are characterized by an intensity range. They are composed of pixels with gray-level values belonging to a given threshold interval (overall luminosity or gray shade). All other pixels are considered to be part of the background. [5], [6]

Thresholding sets all pixels that belong to a range of pixel values, called the threshold interval, to 1 or a user-defined value, and it sets all other pixels in the image to 0 .

\section{Manual Threshold}

The threshold interval in a manual threshold has two userdefined parameters: lower threshold and upper threshold. All pixels that have gray-level values equal to or greater than the lower threshold and equal to or smaller than the upper threshold are selected as pixels belonging to particles in the image. [7]

\section{Automatic Threshold}

Automatic thresholding techniques include:

- Clustering

- Entropy

- InterVariance

- Metric

- Moments

In contrast to manual thresholding, these techniques do not require to set up the lower and upper threshold values. These techniques are well suited for conditions in which the light intensity varies from image to image.

\section{Threshold techniques}

\section{Clustering}

Clustering is the most frequently used automatic thresholding method. It can be used when we need to threshold the image into more than two classes. 
Clustering sorts the histogram of the image within a discrete number of classes corresponding to the number of phases perceived in an image. The gray values are determined, and a barycenter is determined for each class.

This process is repeated until it obtains a value that represents the center of mass for each phase or class.

The threshold value is the pixel value $\mathrm{k}$ for which the following condition is true:

$$
\frac{\mu_{1}+\mu_{2}}{2}=k,
$$

where $\mu_{1}$ is the mean of all pixel values that lie between 0 and $k$, and $\mu_{2}$ is the mean of all the pixel values that lie between $k+1$ and 255 .

\section{Entropy}

Based on a classical image analysis technique, entropy is best for detecting particles that are present in minuscule proportions on the image. For example, this function would be suitable for fault detection.

In this method, the threshold value is obtained by applying information theory to the histogram data. In information theory, the entropy of the histogram signifies the amount of information associated with the histogram. The probability $p(i)$ of occurrence of the gray level $\mathrm{i}$ is represented:

$$
p(i)=\frac{h(i)}{\sum_{i=0}^{N-1} h(i)},
$$

where $h(i)$ represents the number of pixels in the image at each gray level value

The entropy $H$ of a histogram of an image with gray levels in the range $[0, N-1]$ is given by

$$
H=\sum_{i=0}^{N-1} p(i) \log _{2} p(i)
$$

If $k$ is the value of the threshold, then the two entropies represent the measures of the entropy (information) associated with the black and white pixels in the image after thresholding.

Simplified, the threshold value is the pixel value $k$ at which the following expression is maximized:

$$
\begin{aligned}
& H=-\frac{1}{\sum_{i=0}^{k} h(i)^{i=0}} \sum^{k} h(i) \log _{2} h(i)-\frac{1}{\sum_{i=k+1}^{N-1} h(i)^{i=k+1}} \sum^{N-1} h(i) . \\
& \log _{2} h(i)+\log _{2}\left(\sum_{i=0}^{k} h(i) \sum_{i=k+1}^{N-1} h(i)\right)
\end{aligned}
$$

\section{Interclass Variance}

Interclass variance is based on discriminant analysis. An optimal threshold is determined by maximizing the between-class variation with respect to the threshold.
The threshold value is the pixel value $\mathrm{k}$ at which the following expression is maximized:

$$
\sigma_{B}^{2}(k)=\frac{\left[\mu_{T} \omega(k)-\mu(k)\right]^{2}}{\omega(k)[1-\omega(k)]},
$$

where

$$
\begin{aligned}
& \mu(k)=\sum_{i=0}^{k} i p(i), \\
& \mu_{T}=\sum_{i=0}^{N-1} i p(i), \\
& \omega(k)=\sum_{i=0}^{k} p(i),
\end{aligned}
$$

Metric

For each threshold, a value determined by the surfaces representing the initial gray scale is calculated. The optimal threshold corresponds to the smallest value.

The threshold value is the pixel value $\mathrm{k}$ at which the following expression is minimized:

$$
\sum_{i=0}^{k} h(i)\left|\left(i-\mu_{1}\right)\right|+\sum_{i=k+1}^{N-1} h(i)\left|\left(i-\mu_{2}\right)\right|,
$$

where $\mu_{1}$ is the mean of all pixel values in the image that lie between 0 and $k$, and $\mu_{2}$ is the mean of all the pixel values in the image that lie between $k+1$ and 255 .

\section{Moments}

This technique is suited for images that have poor contrast. The moments method is based on the hypothesis that the observed image is a blurred version of the theoretically binary original. The blurring that is produced from the acquisition process, caused by electronic noise or slight defocalization, is treated as if the statistical moments of average and variance were the same for both the blurred image and the original image. This function recalculates a theoretical binary image.

In this method the threshold value is computed in such a way that the moments of the image to be thresholded are preserved in the binary output image.

The $k$-th moment $m$ of an image is calculated as

$$
m_{k}=\frac{1}{n} \sum_{i=0}^{N-1} i^{k} h(i)
$$

where $n$ is the total number of pixels in the image, $N$ represents the total number of gray levels in the image (256 for an 8-bit image).

\section{Human Tissue Measurement}

Virtual instrument was built in collaboration with Jesenius Faculty of Medicine in Martin. Various tissue parameters can be measured for health skin or tissue. The parameters for unhealthy 
skin or tissue can be measured too, with this developed virtual instrument (e.g. psoriasis vulgaris or lichen planus).

The good diagnostic index and parameter are used for derma blood supply evaluation. The derma blood supply is presented by vessels coverage. The goal of the developed virtual instrument is to find the vessels coverage of a searched tissue. The virtual instrument can find vessels coverage for healthy or pathological changed tissue. The region of interest (ROI) can be selected and processed statistically. The vessels coverage is a base for statistical analysis. The vessels are recognized and the ROIs percentage coverage is calculated.

The basic image analysis was performed in NI Vision Assistant Then the script was converted to the LabVIEW [8]. The images of abdominal tissue were used as the base for statistical samples.

The script was preprocessed in NI Vision Assistant after the image analysis. Important color space was extracted and its thresholding was done in this step of processing. Then we created a binary mask together with morphological operators (dilatation, erosion, closing, opening). As these operations are basic for ROI creation we converted the script to LabVIEW. The results of images preprocessing are in Fig. 3.

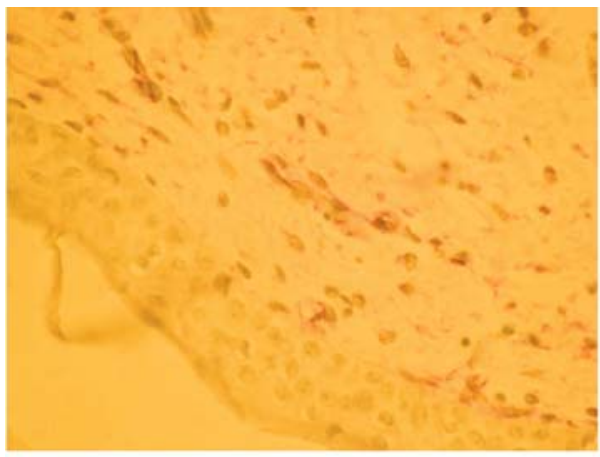

a)

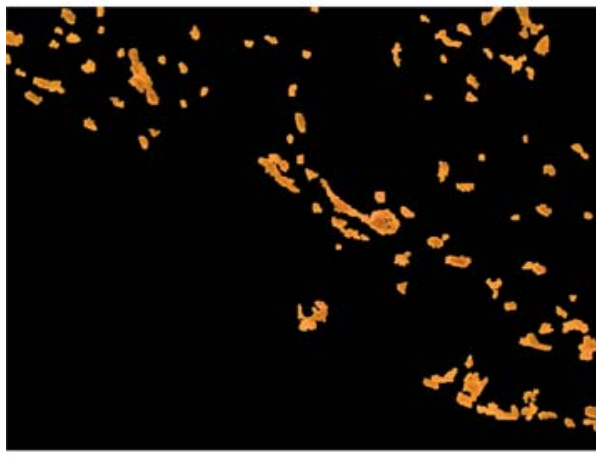

b)

Fig. 3 a) Original image, b) result of preprocessing in NI Vision Assistant

If we want sharpen vessels in this tissue, we have to use a special colorized solution. We can perceive the sharpened vessels as dark red or rusty objects. The specimen background is created from epithelium cells, which are not relevant for our analysis. This fact is shown in Fig. 4.

The tissue samples are scanned as 24 bit RGB color images with JPEG compression. When the images are remapped to single color planes, the histogram can be done for each one of them. Following the histogram distribution and IMAQ tool measurement it can be determined that 8 bit color space Red and Green are approximately identical. The gray levels distribution is different for the color space Blue compared with the color spaces $\mathrm{R}$ and $\mathrm{G}$. The differences are strong between the vessels and the background with cells in the color space B. Therefore the color space B is selected for a threshold. The gray levels values have a closed range, therefore the histogram stretching must be performed to improve gray levels distribution. The threshold is done on stretching image and in this way the threshold is refined.

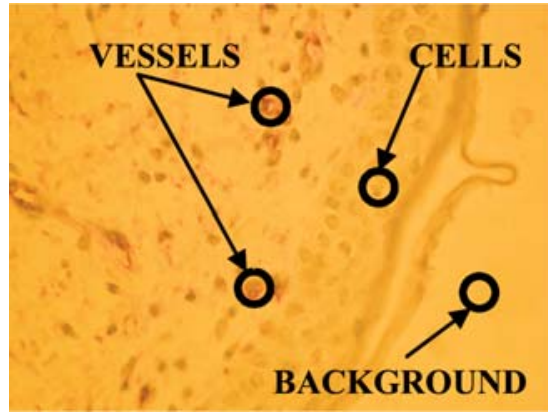

Fig. 4 Abdomen specimen

ROI can be selected manually or automatically. The image centroid is computed in an automatic mode and the ROI size is computed from the original image size (Fig. 5). The ROI centre and dimensions can be changed in manual mode by a user. The other parameters of selected ROI, e.g. threshold value, morphological operators and iterations counts, can be also changed in the manual mode.

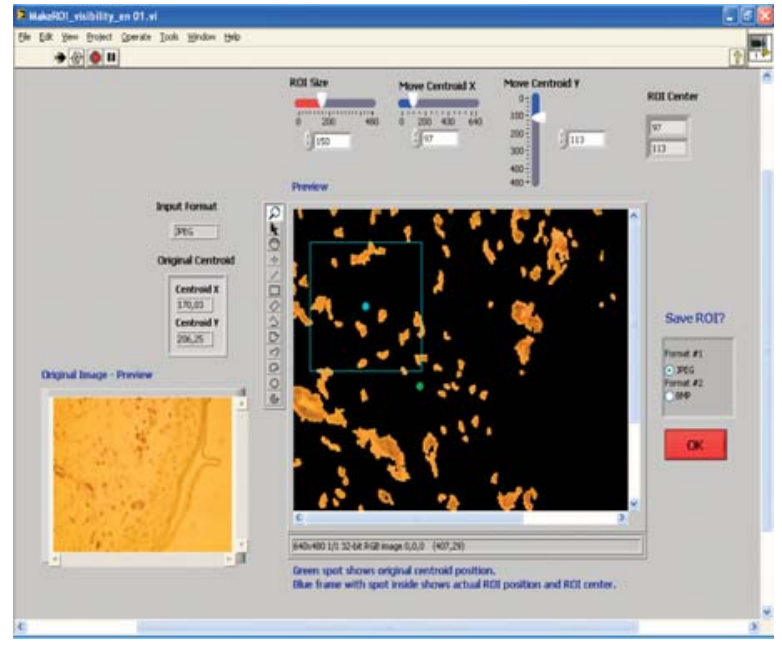

Fig. 5 Application front panel 


\section{Conclusion}

The acquisition system for tissue samples contains intelligent illumination dimming hardware. The hardware automatically regulates halogen lamp lighting for a fast digital camera by means of a measurement card. Regulating parameter for dimmer (PWM duty cycle) is computed from the image features, the histogram distribution and the intensity relations. Dimming helps to preserve optimal light conditions for acquisition system. The optimal light

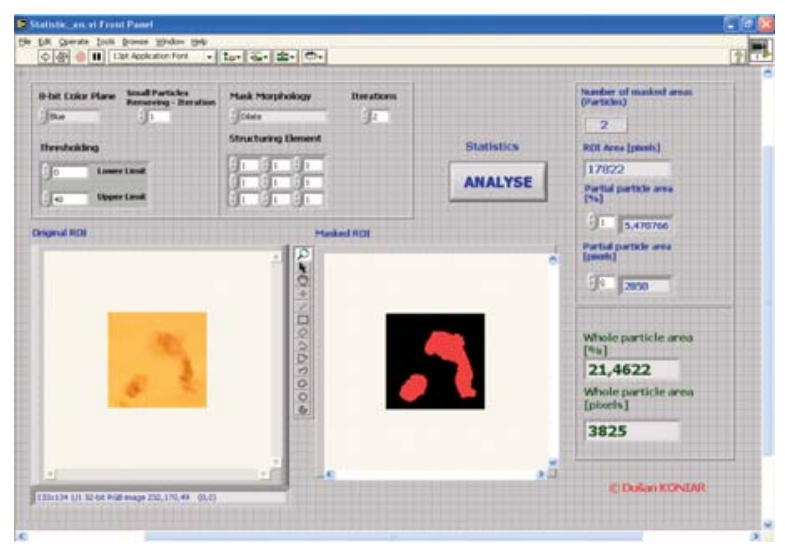

Fig. 6 Statistical analysis of human tissue conditions are important for accurate image processing. The dimmer eliminates abnormal heat generation in microscope condenser for the used high-power halogen lamp.

The derma blood supply is a good diagnostic parameter based on vessels coverage. The vessels coverage for healthy or pathologically changed tissue is searched by the virtual instrument. The consequential analysis can be a help for doctors to diagnose patients. The statistical analysis is realized for objective results comparison (Fig. 6). A ratio between vessels and other tissues (background and cells) can be measured and percentage of ROI vessels coverage can be enumerated. The virtual instrument can objectify tissue samples evaluation.

This virtual instrument can be the first segment in virtual instrumentation integration in medicine or imaging diagnostic methods.

\section{Acknowledgements}

This work has been supported by the grants No. 1/0704/08 and No.1/0087/09 of VEGA grant agency, the project "Centre of Experimental and Clinical Respirology", IMTS code: 26220120004, "Centre of Experimental and Clinical Respirology II", "Measurement of Respiratory Epithelium Cilium Kinematics" and R\&D operational program „Centrum of excellence of power electronics systems and materials for their components", No.OPVaV2008/2.1/01-SORO, ITMS26220120003 funded by the European Community.

\section{References}

[1] FRIVALDSKY, M., DRGONA, P., PRIKOPOVA, A.: Design and Modeling of 200kHz 1,5kW LLC Power Semiconductor Rsonant Converter, IEEE International conference on applied electronics, Pilsen, 2009.

[2] DRGONA, P., BOBEK, V., DOBRUCKY, B., FRIVALDSKY, M.: Performance Analysis of Equation Computation for Real Time Model of PMSM for VHFIM Control of PMSM Position Drive, EDPE 2009, Int. Conference on electrical drives and power electronics.

[3] DUCHON, F., STRENGER, M.: Microsoft Robotics Studio. In: AT\&P Journal Plus, ISSN 1336-5010, No. 1, 2008, pp. 18-23.

[4] JURISICA, L., DUCHON, F.: Mapping in Mobile Robotics. In: Acta Mechanica Slovaca, ISSN 1335-2393, Vol. 12, No. 2-A, 2008, pp. 307-320.

[5] RUSS, J.C.: Image Processing Handbook, CRC Press LLC, 1999

[6] KLINGER, T.: Image Processing with LabVIEW and IMAQ Vision, Prentice Hall, 2003

[7] JURISICA, L., SUROVCIK, T.: Visual System as Evaluation Tool for Moving Mechatronics System (in Slovak). In: Acta Mechanica Slovaca. - ISSN 1335-2393. - Vol. 12, No. 3-B: Modelovanie mechanickych a mechatronickych sustav MMaMS 2008. Cerveny Klastor, 2008, pp. 375-380

[8] ZIDEK J.: Graphical programming in development system LabVIEW(in Czech), Ostrava, 2002.

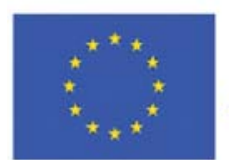

Európska únia
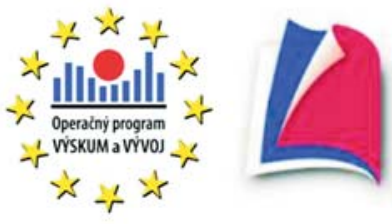

Agentúra

Ministerstva školstva, vedy, výskumu a športu SR pre štrukturálne fondy EÚ

"Podporujeme vyskumne aktivity na Slovensku/Projekt je spolufinancovany zo zdrojov EU." 